\title{
A LIKELY CLOSE-IN LOW-MASS STELLAR COMPANION TO THE TRANSITIONAL DISK STAR HD 142527
}

\author{
Beth Biller $^{1}$, Sylvestre Lacour ${ }^{2}$, Attila Juhász ${ }^{3}$, Myriam Benisty $^{1}$, Gael Chauvin $^{1,4}$, Johan Olofsson $^{1}$, \\ Jörg-Uwe Pott ${ }^{1}$, André Müller ${ }^{1}$, Aurora Sicilia-Aguilar ${ }^{5}$, Mickä̈l Bonnefoy ${ }^{1}$, Peter Tuthill ${ }^{6}$, PhilipPe Thebault ${ }^{2}$, \\ ThOMAS HeNNing ${ }^{1}$, AND AURELIEn CRIDA ${ }^{7}$ \\ ${ }^{1}$ Max-Planck-Institut für Astronomie, Königstuhl 17, 69117 Heidelberg, Germany; biller@mpia.de \\ ${ }^{2}$ LESIA, CNRS/UMR-8109, Observatoire de Paris, UPMC, Université Paris Diderot, 5 place Jules Janssen, 92195 Meudon, France \\ ${ }^{3}$ Leiden Observatory, Leiden University, P.O. Box 9513, 2300 RA Leiden, The Netherlands \\ ${ }^{4}$ UJF-Grenoble 1/CNRS-INSU, Institut de Planétologie et dAstrophysique de Grenoble (IPAG) UMR 5274, Grenoble, F-38041, France \\ ${ }^{5}$ Departamento de Física Teórica, Facultad de Ciencias, Universidad Autónoma de Madrid, 28049 Cantoblanco, Madrid, Spain \\ ${ }^{6}$ School of Physics, University of Sydney, NSW 2006, Australia \\ ${ }^{7}$ Université de Nice - Sophia antipolis / C.N.R.S. / Observatoire de la Côte d'Azur, Laboratoire Lagrange (UMR 7293), Boulevard de l'Observatoire, \\ B.P. 422906304 NICE cedex 04, France \\ Received 2012 April 27; accepted 2012 June 6; published 2012 June 26
}

\begin{abstract}
With the uniquely high contrast within $0^{\prime} .1\left(\Delta \operatorname{mag}\left(L^{\prime}\right)=5-6.5 \mathrm{mag}\right)$ available using Sparse Aperture Masking with NACO at Very Large Telescope, we detected asymmetry in the flux from the Herbig Fe star HD 142527 with a barycenter emission situated at a projected separation of $88 \pm 5$ mas $(12.8 \pm 1.5 \mathrm{AU}$ at $145 \mathrm{pc})$ and flux ratios in $H, K$, and $L^{\prime}$ of $0.016 \pm 0.007,0.012 \pm 0.008$, and $0.0086 \pm 0.0011$, respectively ( $3 \sigma$ errors), relative to the primary star and disk. After extensive closure-phase modeling, we interpret this detection as a close-in, low-mass stellar companion with an estimated mass of $\sim 0.1-0.4 M_{\odot}$. HD 142527 has a complex disk structure, with an inner gap imaged in both the near and mid-IR as well as a spiral feature in the outer disk in the near-IR. This newly detected low-mass stellar companion may provide a critical explanation of the observed disk structure.
\end{abstract}

Key words: brown dwarfs - circumstellar matter - planetary systems - stars: emission-line, Be - stars: low-mass

Online-only material: color figures

\section{INTRODUCTION}

Transition disks may trace a key step in the process of forming planets and dissipating primordial stellar disks. Transition disks are primordial disks characterized by weak mid-IR emission (at $\sim 15 \mu \mathrm{m}$ ) relative to the Taurus median spectral energy distribution (i.e., the median SED of primordial disks in the young ( $<2 \mathrm{Myr}$ ) Taurus star-forming region; Najita et al. 2007). A number of transition disks possess gaps either posited from SED studies or directly imaged (Andrews et al. 2011; Fukagawa et al. 2006; Pott et al. 2010; Brown et al. 2009) which may be due to clearing of dust by a forming planet or brown dwarf and thus may produce the observed mid-IR deficit. Transition disks have therefore been popular targets for high-contrast high-resolution imaging planet searches.

While transition disks have previously been searched for planets using high contrast imaging techniques (e.g., adaptive optics and coronagraphy), most of these techniques currently do not extend to the inner 0 '. 1 , which corresponds to the crucial planetforming regions $(\sim 10 \mathrm{AU})$ of the disk for objects at distances $>100 \mathrm{pc}$. Only interferometric techniques possess the resolution to reveal these inner regions (see, e.g., Pott et al. 2010). The interferometric technique of Sparse Aperture Masking (SAM) uniquely allows us to both probe the inner 0 .' 1 of transitional disks and reach high contrasts of $\Delta \operatorname{mag}\left(L^{\prime}\right)=5-6.5$ mag. Probing this region enables us to understand how planets form in their native disk and how they impact the surrounding material. Indeed, observations using SAM have already yielded two planetary or brown dwarf candidate companions to transition disk stars (Huélamo et al. 2011; Kraus \& Ireland 2012).

The transition disk star HD 142527 is a Herbig Fe star with spectral type of F6 IIIe (Houk 1978; Henize 1976; Waelkens et al. 1996). HD 142527 notably possesses a very complex and interesting disk that has long been posited as a possible site of planet formation. Recent SED modeling and VISIR imaging suggests a disk gap from 30 to 130 AU (Verhoeff et al. 2011). The outer edge of the gap as well as a spiral feature in the outer disk have been imaged in the near-IR (Fukagawa et al. 2006). Fukagawa et al. (2006) also find an offset of 20 AU between the star center and disk center, which they posit is caused by an unseen eccentric binary companion. Baines et al. (2006) note this system as a possible (but unconfirmed) binary detection from spectroastrometry. The disk of HD 142527 also possesses an extremely high fraction of crystalline silicates, possibly formed by a massive companion inducing spiral density waves in the disk material (van Boekel et al. 2004). Here we report the discovery of a likely close-in, low-mass stellar companion $\left(12.8 \pm 1.5 \mathrm{AU}\right.$ at $145 \mathrm{pc}$ and flux ratios in $H, K$, and $L^{\prime}$ of $0.016 \pm 0.007,0.012 \pm 0.008$, and $0.0086 \pm 0.0011$, respectively) to this star. This is the first confirmation of the binarity of HD 142527 and may provide a critical explanation of the observed disk structure.

\section{STELLAR PARAMETERS}

Hipparcos measurements for HD 142527 yield a distance of $230_{-40}^{+70} \mathrm{pc}$ (van Leeuwen 2007). Alternately, HD 142527 has been associated with both the Sco OB-2 association (Acke $\&$ van den Ancker 2004) and Upper Centaurus Lupus (de Zeeuw et al. 1999; Teixeira et al. 2000). Membership in either association (indeed, both are part of the larger Sco-Cen association) place HD 142527 at a distance of 140-145 pc (de Zeeuw et al. 1999) and an age of 2-10 Myr. We consider the evidence of association within Sco-Cen to be very strong and thus adopt a distance of $145 \pm 15 \mathrm{pc}$, which still lies within $2 \sigma$ of the rather uncertain Hipparcos measurement. 
Table 1

Properties of the HD 142527 AB System

\begin{tabular}{|c|c|c|}
\hline & Primary + Disk & Secondary \\
\hline Distance & \multicolumn{2}{|c|}{$145 \pm 15 \mathrm{pc}^{\mathrm{a}}$} \\
\hline Age & \multicolumn{2}{|c|}{$5_{-3}^{+8} \mathrm{Myr}^{\mathrm{a}}$} \\
\hline Proper motion $\left(\mu_{\alpha}, \mu_{\delta}\right)$ & \multicolumn{2}{|c|}{$(-11.19 \pm 0.93,-24.46 \pm 0.79) \mathrm{mas} \mathrm{yr}^{-1 b}$} \\
\hline Separation: UT 2012 March 11 & \multicolumn{2}{|c|}{$88 \pm 5 \operatorname{mas}(12.8 \pm 1.5 \mathrm{AU})$} \\
\hline Position angle: UT 2012 March 11 & \multicolumn{2}{|c|}{$133.3 \pm 2.5$} \\
\hline Flux ratio in $H$ & $\ldots$ & $0.016 \pm 0.007$ \\
\hline Flux ratio in $K s$ & $\ldots$ & $0.012 \pm 0.008$ \\
\hline Flux ratio in $L^{\prime}$ & $\ldots$ & $0.0086 \pm 0.001$ \\
\hline$H(\mathrm{mag})$ & $5.94^{\mathrm{c}}$ & $10.5 \pm 0.2$ \\
\hline$K s$ (mag) & $5.20^{\mathrm{c}}$ & $10.0 \pm 0.3$ \\
\hline$L^{\prime}(\mathrm{mag})$ & $3.89^{\mathrm{c}}$ & $9.1 \pm 0.1$ \\
\hline$H-K s(\mathrm{mag})$ & 0.74 & $0.5 \pm 0.4$ \\
\hline$K s-L^{\prime}(\mathrm{mag})$ & 1.31 & $0.9 \pm 0.3$ \\
\hline$M_{H}(\mathrm{mag})$ & $0.2 \pm 0.2$ & $4.8 \pm 0.3$ \\
\hline$M_{K_{s}}(\mathrm{mag})$ & $-0.6 \pm 0.2$ & $4.2 \pm 0.3$ \\
\hline$M_{L^{\prime}}(\mathrm{mag})$ & $-1.9 \pm 0.2$ & $3.3 \pm 0.2$ \\
\hline Spectral type & F6IIIe & $\ldots$ \\
\hline Estimated mass & $2.2 \pm 0.3 M_{\odot}^{\mathrm{a}}$ & $0.1-0.4 M_{\odot}$ \\
\hline
\end{tabular}

Notes.

a Verhoeff et al. (2011).

b van Leeuwen (2007).

${ }^{c}$ Malfait et al. (1998).

Verhoeff et al. (2011) obtain a stellar luminosity of $15 \pm$ $2 L_{\odot}$ from comparison to Kurucz (1991) photospheric models for the object spectral type of F6 III. They correct the stellar luminosity to $20 \pm 2 L_{\odot}$ after taking into account a modeldependent gray extinction component of the disk and halo and obtain updated stellar parameters by comparing the position of this star in the Hertzsprung-Russell diagram to the pre-mainsequence evolution tracks of Siess et al. (2000). Here, we adopt the stellar parameters from Verhoeff et al. (2011) - specifically, a stellar mass of $2.2 \pm 0.3 \mathrm{M}_{\odot}$ and an age of $5_{-3}^{+8}$ Myr consistent with membership in the Sco OB-2 association (Table 1).

\section{OBSERVATIONS AND DATA REDUCTION}

HD 142527 was observed on 2012 March 10 with Very Large Telescope (VLT) NACO ${ }^{8}$ Observations were taken in the $H, K$, and $L^{\prime}$ bands $\left(\lambda_{\mathrm{L}^{\prime}}=3.80 \pm 0.31 \mu \mathrm{m}\right)$ using the "7 holes" aperture mask and the IR wavefront sensor (WFS). The observing $\log$ is presented in Table 2. The target was observed in each band for 30 minutes to $2 \mathrm{hr}$.

The use of the "7 holes" (C7-892; Tuthill et al. 2010) aperture mask transforms the telescope into a Fizeau interferometer. The point-spread function is a complex superposition of fringes at given spatial frequencies. In specific cases, pupil-masking can outperform more traditional differential imaging for a number of reasons (Tuthill et al. 2006; Lacour et al. 2011b). First, the masks are designed to have nonredundant array configurations that permit phase deconvolution; slowly moving optical aberrations not corrected by the AO can be accurately calibrated. Second, the mask primarily rejects baselines with low spatial frequency and passes proportionately far more baselines with higher $\lambda / B$ (where $B$ is baseline length) resolution than does an orthodox fully filled pupil. Third, high-fidelity recovery of phase information allows "super resolution," with a marginal loss of dynamic range up to $\lambda / 2 D$ (where $D$ is the mirror diameter). The principal drawback is a loss in throughput so that photon and

\footnotetext{
8 Program ID: 088.C-0691(A)
}

Table 2

HD 142527 2012-03-11 (UT) Observation log

\begin{tabular}{|c|c|c|c|c|}
\hline Target & UT time & Band & $\begin{array}{l}\text { DIT } \\
(\mathrm{ms})\end{array}$ & NDIT \\
\hline HD 142527 & 2012-03-11T06:02:25.1402 & $L^{\prime}$ & 120 & 330 \\
\hline HD 142695 & 2012-03-11T06:37:33.3990 & $L^{\prime}$ & 120 & 330 \\
\hline HD 142527 & 2012-03-11T06:46:55.5591 & $L^{\prime}$ & 120 & 330 \\
\hline HD 142384 & 2012-03-11T06:57:19.0879 & $L^{\prime}$ & 120 & 330 \\
\hline HD 142527 & 2012-03-11T07:07:34.3389 & $L^{\prime}$ & 120 & 330 \\
\hline HD 144350 & 2012-03-11T07:18:46.5699 & $L^{\prime}$ & 120 & 330 \\
\hline HD 142527 & 2012-03-11T07:32:05.3348 & $L^{\prime}$ & 120 & 330 \\
\hline HD 142695 & 2012-03-11T07:41:37.5524 & $L^{\prime}$ & 120 & 330 \\
\hline HD 142527 & 2012-03-11T07:51:14.5767 & $L^{\prime}$ & 120 & 330 \\
\hline HD 142527 & 2012-03-11T08:13:19.5884 & $K s$ & 109 & 360 \\
\hline HD 142384 & 2012-03-11T08:27:00.5782 & $K s$ & 109 & 360 \\
\hline HD 142527 & 2012-03-11T08:42:30.3669 & $K s$ & 109 & 360 \\
\hline HD 142695 & 2012-03-11T08:53:16.9986 & $K s$ & 109 & 360 \\
\hline HD 142527 & 2012-03-11T09:03:59.4995 & $K s$ & 109 & 360 \\
\hline HD 142527 & 2012-03-11T09:23:19.1784 & $H$ & 100 & 360 \\
\hline HD 142384 & 2012-03-11T09:34:21.3448 & $H$ & 100 & 360 \\
\hline HD 142527 & 2012-03-11T09:44:44.1641 & $H$ & 100 & 360 \\
\hline
\end{tabular}

detector noise can affect the signal-to-noise ratio even where targets are reasonably bright for the AO system. The effective field of view of SAM is determined by the shortest baseline so that the technique is not competitive at separations that are greater than several times the formal diffraction limit. For SAM observations with the "7 holes" mask with VLT NACO, the field of view is 300 mas in the $H$ band, 400 mas in the $K$ band, and 600 mas in the $L$ band. For more details on the SAM mode, see, e.g., Lacour et al. (2011a) and Tuthill et al. (2010).

The HD 142527 observations were processed with both the Sydney FFT and Observatoire de Paris SAMP pipelines (Tuthill et al. 2000; Lacour et al. 2011a). Both the $\chi^{2}$ map and the phase in the UV plane (extracted from the closure phases; see Huélamo et al. 2011) show an asymmetry typical of point sources (at the 

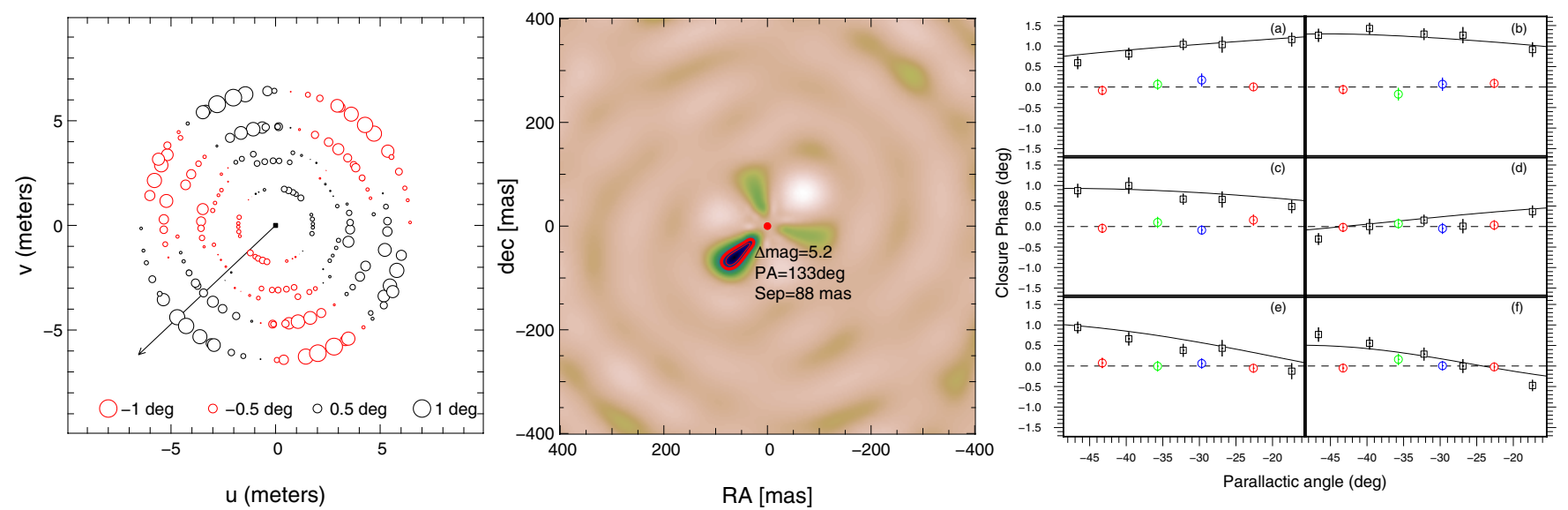

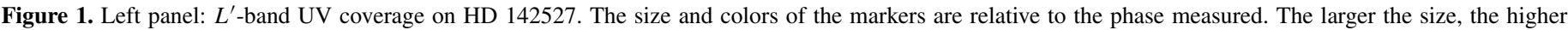

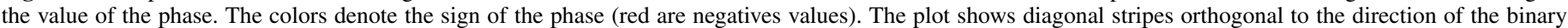

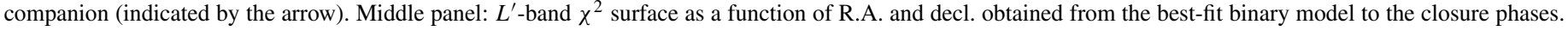

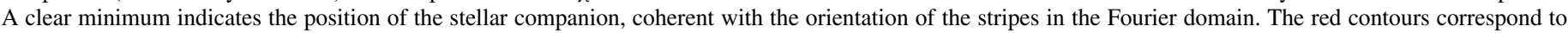

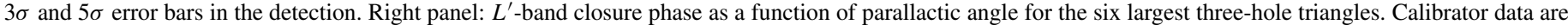

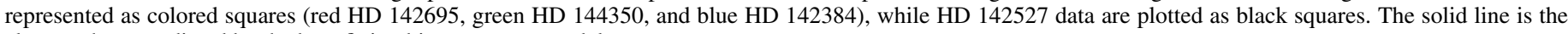
closure phase predicted by the best-fitting binary system model.

(A color version of this figure is available in the online journal.)

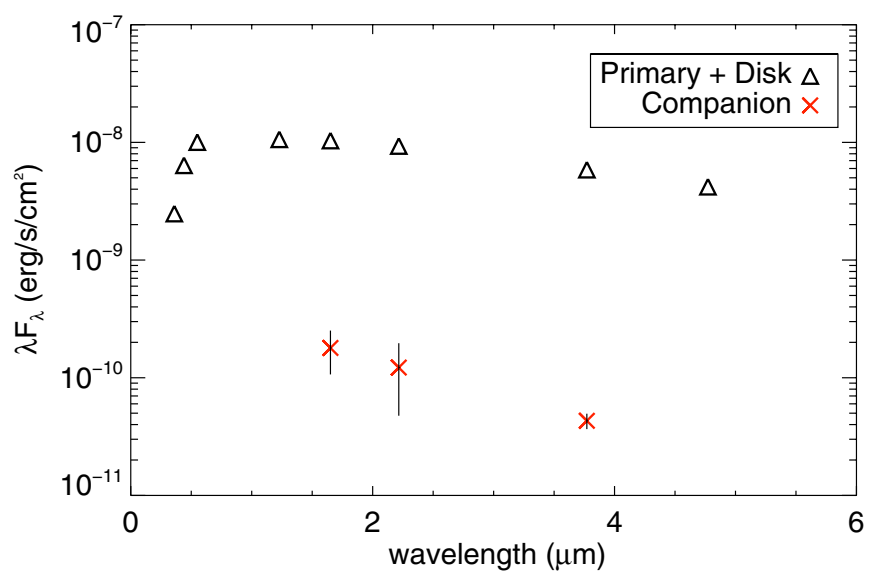

Figure 2. SED for HD 142527 as well as companion fluxes in the same bandpasses. SED data points are drawn from the photometry of Malfait et al. (1998).

(A color version of this figure is available in the online journal.)

resolution of the telescope; see left panel of Figure 1). Therefore, we fitted the closure phases with a model of two point-like objects (the star and a companion of lesser flux). In all three bands, the best-fit model to the closure phases shows a pointlike asymmetry at $88 \pm 5$ mas from the central star with flux ratios in $H, K$, and $L^{\prime}$ of $0.016 \pm 0.007,0.012 \pm 0.008$, and $0.0086 \pm 0.0011$, respectively ( $3 \sigma$ errors), relative to the primary star and disk. The $\chi^{2}$ map of the $L^{\prime}$-band data is shown in the right panel of Figure 1. The fit on several of the 35 closure phases (triangle) is plotted in Figure 2 ( $L^{\prime}$-band data).

\section{RESULTS}

\subsection{Photometry}

We adopt the $H K L$ magnitudes from Malfait et al. (1998), since no $L$-band data are available from the Two Micron All Sky Survey (2MASS). We note, however, that there is a significant divergence between the reported 2MASS photometry and the Malfait et al. (1998) photometry. The 2MASS photometry is brighter than the Malfait et al. (1998) photometry by
0.1-0.3 mag, which may suggest variability for this system. No errors are provided for the Malfait et al. (1998) photometry; we assume error bars are similar to the 2MASS photometry.

Raw photometry for this system is comprised of light from three components-primary star, secondary companion, and disk. Some portion of the disk (the "outer disk") lies outside of the SAM field of view. From the SED model of Verhoeff et al. (2011) we estimate that the outer disk comprises $\sim 10 \%$ or less of the total system flux at $H K L^{\prime}$. Thus, we do not correct the raw photometry to remove the outer disk component. Magnitudes in $H, K$, and $L^{\prime}$ for the star+disk are presented in Table 1 .

The detected companion has flux ratios in $H, K$, and $L^{\prime}$ of $0.016 \pm 0.007,0.012 \pm 0.008$, and $0.0086 \pm 0.0011$, respectively ( $3 \sigma$ errors). To account for errors in the initial photometry as well as our measured SAM flux ratio, we adopt a Monte Carlo approach. We simulated an ensemble of $10^{6}$ observations per band, with photometry and flux ratios drawn from Gaussian distributions centered on the measured values and with $\sigma$ drawn from the reported errors. The apparent magnitude in each band is given as the median of this ensemble, with error bars drawn from the standard deviation of the same ensemble. Thus, the measured flux ratios and primary star photometry correspond to apparent magnitudes of $10.5 \pm 0.2$, $10.0 \pm 0.3$, and $9.1 \pm 0.1$ in $H, K$, and $L^{\prime}$ (Table 1 , in CIT bandpasses; Elias et al. 1982). The companion appears anomalously bright in $L^{\prime}$. While the $H-K$ colors are similar to what would be expected for a young red companion, $K-L^{\prime}$ $\sim 0.9$ mag, diverging significantly from the expected value of $\sim 0.4$ mag (Baraffe et al. 1998). Companion fluxes in these bandpasses, along with the full SED for the system are plotted in Figure 2.

We employed a similar Monte Carlo approach in converting from apparent to absolute magnitudes. For our ensemble of $10^{6}$ simulated objects, we simulate corresponding distances drawn from a Gaussian centered at $145 \mathrm{pc}$ and with $\sigma$ of $15 \mathrm{pc}$. Absolute magnitudes are also reported in Table 1.

\subsection{Probability of Chance Alignment}

We estimated the likelihood that this companion is an unrelated background or foreground object using source counts 

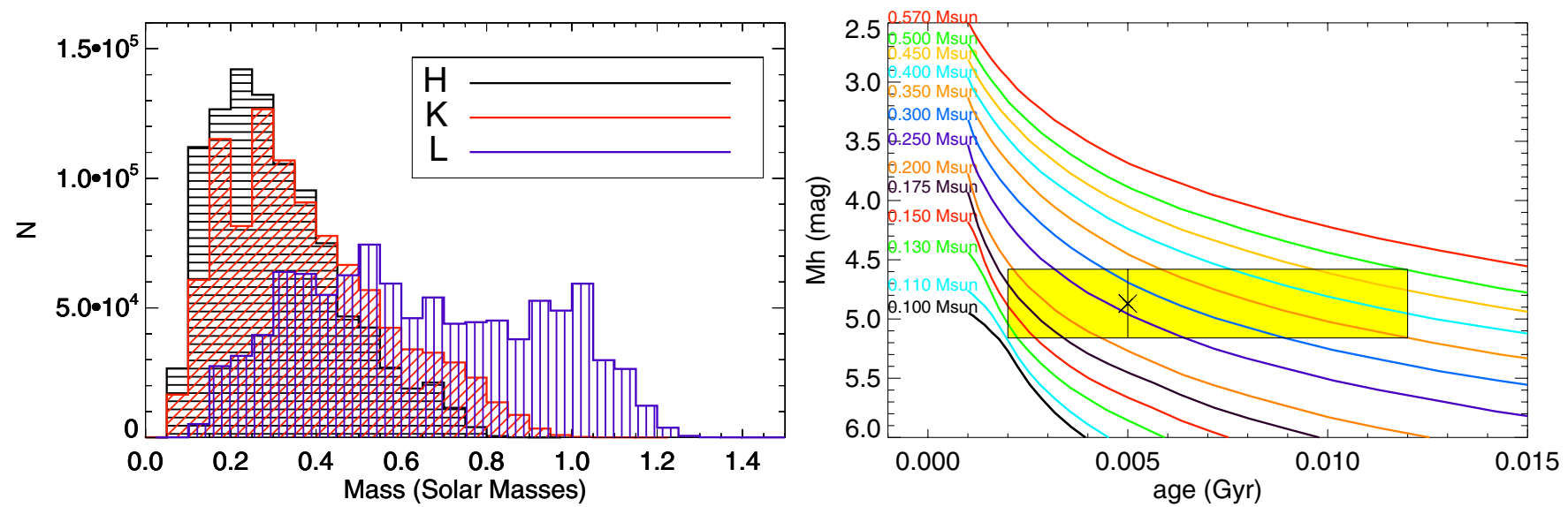

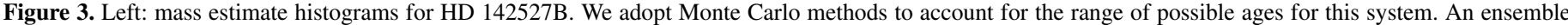

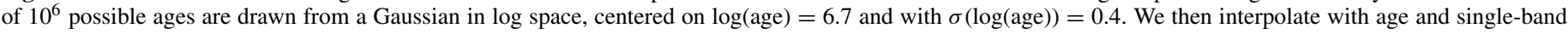

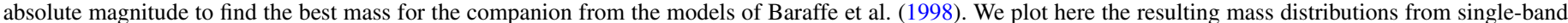

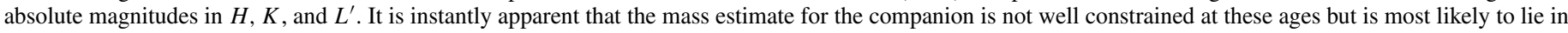

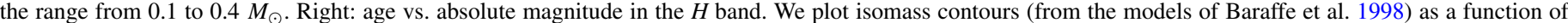

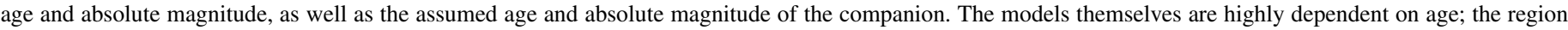
constrained by the observations (yellow rectangle) is consistent with companion masses from 0.1 to $0.4 M_{\odot}$.

(A color version of this figure is available in the online journal.)

from the 2MASS survey. Within a $1 \mathrm{deg}$ radius of the primary, 2MASS detects 1918 objects with $H$ of 10.7 mag or brighter and 1505 objects with $K s$ of 10.0 mag or brighter. Thus, adopting the approach of Brandner et al. (2000), in particular their Equation (1), we estimate the probability of finding an unrelated source at least as bright as the observed companion within $0^{\prime \prime} .088$ of the primary to be $\sim 1.1 \times 10^{-6}$ in the $H$ band and $\sim 8.3 \times 10^{-7}$ in $K s$. We also considered simulated stellar populations along this line of sight (Galactic latitude and longitude of 335.6549 , +08.4804 ) using the Besançon Galactic population synthesis models (Robin et al. 2003). This line of sight is directly into the Galactic bulge, so the models yield 882 background sources per square degree that are brighter than $K=10.5 \mathrm{mag}$. However, the chances of finding one of these within 0 '.088 of the primary are still vanishingly small— $\sim 1.6 \times 10^{-6}$ —and these objects are predominantly $\mathbf{M}$ giant stars, with considerably bluer expected colors (for an M5III star, $H-K=0.29 \mathrm{mag}$ and $K-L^{\prime}=$ 0.22 mag; Tokunaga 2000) than measured for the detected companion. It is therefore extraordinarily unlikely that the companion is unrelated to the primary, although proper motion confirmation in a year will be necessary to finally determine this.

\subsection{Mass Estimate}

Estimated masses for both system components are highly dependent on adopted age. Certainly the HD 142527 system is quite young, but whether it is 1 Myr or 10 Myr makes a critical difference in the mass estimate for the faint companion. Here, we adopt a similar age range as Verhoeff et al. (2011), which is dependent on membership in the Sco OB-2 association.

We again adopt Monte Carlo methods to account for the range of possible ages for this system. An ensemble of $10^{6}$ possible ages are drawn from a Gaussian in log space, centered on $\log ($ age $)=6.7$ and with $\sigma(\log ($ age $))=0.4$. We then interpolate with age and single-band absolute magnitude to find the best mass for the companion from the models of Baraffe et al. (1998). The resulting mass distributions are presented as histograms in Figure 3. It is apparent that the mass estimate for the companion is not well constrained at these ages but is most likely to lie in the range from 0.1 to $0.4 M_{\odot}$. We estimate a best mass estimate for each band of $0.28 \pm 0.15 M_{\odot}, 0.34 \pm 0.19 M_{\odot}$, and $0.60 \pm 0.29 M_{\odot}$ in $H, K$, and $L^{\prime}$ respectively. However, the mass distributions from our Monte Carlo simulations are highly non-Gaussian, with significant probability to find considerably higher companion masses. All mass estimates are within $2 \sigma$ of each other, but the $L^{\prime}$-band mass estimate is particularly high and we note that the companion appears anomalously bright in $L^{\prime}$. While the $H-K$ colors are similar to what would be expected for a young red companion, $K-L^{\prime} \sim 0.9$ mag, considerably divergent from the expected value of $0.4 \mathrm{mag}$. We thus do not attempt to estimate spectral type using $H-K$ and $K-L^{\prime}$ colors. The models of Siess et al. (2000) yield a similar mass range for the companion of $0.1-0.4 M_{\odot}$ for ages of 2-12 Myr. The models themselves are highly dependent on age; to illustrate this, we plot isomass contours (from the models of Baraffe et al. 1998) as a function of absolute $H$ magnitude and age in Figure 3.

\subsection{Constraints on the Orbit}

We estimate the semimajor axis of HD 142527B's orbit from its observed separation. Assuming a uniform eccentricity distribution between $0<\mathrm{e}<1$ and random viewing angles, Dupuy et al. (2010) compute a median correction factor between projected separation and semimajor axis of $1.10_{-0.36}^{+0.91}(68.3 \%$ confidence limits). Using this, we derive a semimajor axis of $14_{-5}^{+12}$ AU. While the mass estimate for the companion is quite uncertain, we adopt the $K$-band value as characteristic and adopt a total system mass of $2.54 \pm 0.35 M_{\odot}$. Our derived semimajor axis estimate corresponds to an orbital period estimate of $33_{-18}^{+42}$ years.

After a year, we expect up to 20 mas of orbital motion on the sky for the companion, which is easily detectable with SAM. The degree of motion observed will put important constraints on the mass of the companion (after adopting an estimate of the mass of the primary) and will provide a key data point for an eventual dynamical mass determination for this system. Assuming a coverage of one third of an orbit is necessary for a good orbital determination (Dupuy et al. 2010); such a determination may be possible in 10 year timescales for 
this system. HD 142527A has a measured proper motion of $-11.19 \pm 0.93$ mas in R.A. and $-24.46 \pm 0.79$ in decl.—about $\sim 20$ mas on-sky motion in a year, with $3 \sigma$ astrometric errors of $\sim 5$ mas. Thus, we will also be able to completely rule out the extremely unlikely case that the companion is an unrelated background object.

\section{DISCUSSION}

The existence of an inner binary has been predicted for HD 142527 (Fukagawa et al. 2006; Baines et al. 2006) but this is the first confirmation of the binary companion. The inner binary likely explains the $20 \mathrm{AU}$ offset observed by Fukagawa et al. (2006) between the primary and the disk center (Pichardo et al. 2008; Nelson 2003).

The discovery of the inner binary provides an important update for modeling efforts of the structure of the inner disk. The modeling efforts of Verhoeff et al. (2011) find a flat, dusty inner disk from 0.3 to $30 \mathrm{AU}$ (the outer radius supported as well by marginally resolved VISIR imaging), an optically thin halo from 0.3 to $30 \mathrm{AU}$, and an outer disk starting at $130 \mathrm{AU}$. The current projected separation for the companion, $\sim 13 \mathrm{AU}$, places it right inside the modeled inner disk! Thus, it is likely that the companion may produce a cleared ring within the inner disk. Models of the physical properties of disks around eccentric stellar binaries often show entirely cleared inner disks (Pichardo et al. 2008), which may not be the case here.

The HD 142527 disk is notable for being comparably bright or even brighter than the primary star at infrared wavelengths. At the $H$ and $K$ bands, the star is as bright or brighter than the disk, whereas at the $L$ band, the disk is considerably brighter than the star. We consider all components of the Verhoeff et al. (2011) SED model in deriving photometry for the companion. However, if a portion of the inner disk does not contribute to the brightness of the central source, this may be the reason why our $L^{\prime}$-band flux measurement for the companion is anomalously bright. Alternatively, the very bright measured $L^{\prime}$-band magnitude may suggest that the secondary has its own small circumstellar disk (with possible accretion onto the secondary) or that the relatively massive secondary may produce local disk heating. Transient heating caused by the secondary inducing spiral shock waves in the disk material could also be the source of the high fraction of crystalline silicates found at large radii in this disk (van Boekel et al. 2004).

The large cavity observed in the HD 142527 disk may be the signature of an unseen companion interacting with the inner binary. A binary + planet system can open a much larger gap than can be formed by the binary by itself (Nelson 2003; Kley et al. 2008; Kley \& Nelson 2008, 2012). Nelson (2003) note that in the case of an eccentric inner binary system, the circumbinary disk itself can become eccentric, ending the inner migration of the planet and producing a stable orbital configuration. This seems a likely explanation of the observed disk structure in the HD 142527 system, especially the wide gap within $130 \mathrm{AU}$, but must be confirmed by continued orbital monitoring of the binary system to confirm that it is indeed in an eccentric orbit. Followup SAM observations are thus absolutely critical for this system and are still possible within the lifespan of NACO. Eventually, a dynamical mass can be determined for this system, perhaps in 10 year timescales and monitoring on 1-2 year timescales may help confirm the eccentricity of the orbit (see, e.g., Biller et al. 2010).

\section{CONCLUSIONS}

We detect a likely close companion to HD 142527 with separation of $88 \pm 5$ mas $(12.8 \pm 1.5 \mathrm{AU}$ at $145 \mathrm{pc})$ and flux ratios in $H, K$, and $L^{\prime}$ of $0.016 \pm 0.007,0.012 \pm 0.008$, and $0.0086 \pm 0.0011$, respectively ( $3 \sigma$ errors), relative to the primary star and inner disk. The companion is consistent with mass estimates of $0.1-0.4 M_{\odot}$ from the models of Baraffe et al. (1998). However, continued orbital monitoring will be necessary to provide more accurate mass estimates, as model masses contain significant uncertainties at these young ages. The inner binary likely explains the 20 AU offset observed by Fukagawa et al. (2006) between the primary and the disk center (Pichardo et al. 2008; Nelson 2003). Additionally, the large cavity observed in the HD 142527 may be the signature of an unseen planet interacting with the inner binary.

Based on observations made with ESO Telescopes at the La Silla Paranal Observatory under programme ID 088.C-0691. We gratefully acknowledge the support of the ESO VLT UT4 staff as well as useful conversations with Roy van Boekel and Jeroen Bouwman.

\section{REFERENCES}

Acke, B., \& van den Ancker, M. E. 2004, A\&A, 426, 151

Andrews, S. M., Wilner, D. J., Espaillat, C., et al. 2011, ApJ, 732, 42

Baines, D., Oudmaijer, R. D., Porter, J. M., \& Pozzo, M. 2006, MNRAS, 367, 737

Baraffe, I., Chabrier, G., Allard, F., \& Hauschildt, P. H. 1998, A\&A, 337, 403

Biller, B. A., Liu, M. C., Wahhaj, Z., et al. 2010, ApJ, 720, L82

Brandner, W., Zinnecker, H., Alcalá, J. M., et al. 2000, AJ, 120, 950

Brown, J. M., Blake, G. A., Qi, C., et al. 2009, ApJ, 704, 496

de Zeeuw, P. T., Hoogerwerf, R., de Bruijne, J. H. J., Brown, A. G. A., \& Blaauw, A. 1999, AJ, 117, 354

Dupuy, T. J., Liu, M. C., Bowler, B. P., et al. 2010, ApJ, 721, 1725

Elias, J. H., Frogel, J. A., Matthews, K., \& Neugebauer, G. 1982, AJ, 87, 1029

Fukagawa, M., Tamura, M., Itoh, Y., et al. 2006, ApJ, 636, L153

Henize, K. G. 1976, ApJS, 30, 491

Houk, N. 1978, Michigan Catalogue of Two-dimensional Spectral Types for the HD Stars (Ann Arbor, MI: Univ. Michigan)

Huélamo, N., Lacour, S., Tuthill, P., et al. 2011, A\&A, 528, L7

Kley, W., \& Nelson, R. P. 2008, A\&A, 486, 617

Kley, W., \& Nelson, R. P. 2012, ARA\&A, submitted (arXiv:1203.1184)

Kley, W., Papaloizou, J. C. B., \& Ogilvie, G. I. 2008, A\&A, 487, 671

Kraus, A. L., \& Ireland, M. J. 2012, ApJ, 745, 5

Kurucz, R. L. 1991, in Precision Photometry: Astrophysics of the Galaxy, ed.

A. G. D. Philip, A. R. Upgren, \& K. A. Janes (Schenectady, NY: Davis Press), 27

Lacour, S., Tuthill, P., Amico, P., et al. 2011a, A\&A, 532, A72

Lacour, S., Tuthill, P., Ireland, M., Amico, P., \& Girard, J. 2011b, The Messenger, 146,18

Malfait, K., Bogaert, E., \& Waelkens, C. 1998, A\&A, 331, 211

Najita, J. R., Strom, S. E., \& Muzerolle, J. 2007, MNRAS, 378, 369

Nelson, R. P. 2003, MNRAS, 345, 233

Pichardo, B., Sparke, L. S., \& Aguilar, L. A. 2008, MNRAS, 391, 815

Pott, J.-U., Perrin, M. D., Furlan, E., et al. 2010, ApJ, 710, 265

Robin, A. C., Reylé, C., Derrière, S., \& Picaud, S. 2003, A\&A, 409, 523

Siess, L., Dufour, E., \& Forestini, M. 2000, A\&A, 358, 593

Teixeira, R., Ducourant, C., Sartori, M. J., et al. 2000, A\&A, 361, 1143

Tokunaga, A. 2000, in Allen's Astrophysical Quantities, ed. A. N. Cox (New York: Springer), 152

Tuthill, P., Lacour, S., Amico, P., et al. 2010, Proc. SPIE, 7735, 773510

Tuthill, P., Lloyd, J., Ireland, M., et al. 2006, Proc. SPIE, 6272, 62723A

Tuthill, P. G., Monnier, J. D., \& Danchi, W. C. 2000, Proc. SPIE, 4006, 491

van Boekel, R., Min, M., Leinert, C., et al. 2004, Nature, 432, 479

van Leeuwen, F. 2007, A\&A, 474, 653

Verhoeff, A. P., Min, M., Pantin, E., et al. 2011, A\&A, 528, A91

Waelkens, C., Waters, L. B. F. M., de Graauw, M. S., et al. 1996, A\&A, 315 , L245 